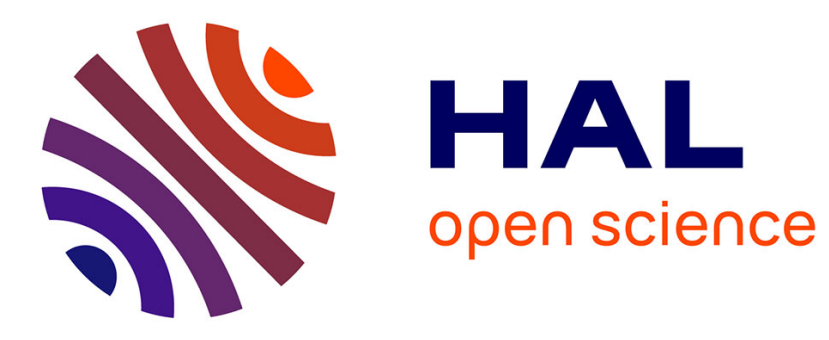

\title{
Analytical Fiber ODF Reconstruction in 3D Polarized Light Imaging: Performance Assessment
}

Abib Alimi, Samuel Deslauriers-Gauthier, Felix Matuschke, Daniel Schmitz, Markus Axer, Rachid Deriche

\section{- To cite this version:}

Abib Alimi, Samuel Deslauriers-Gauthier, Felix Matuschke, Daniel Schmitz, Markus Axer, et al.. Analytical Fiber ODF Reconstruction in 3D Polarized Light Imaging: Performance Assessment. ISBI 2019 - IEEE International Symposium on Biomedical Imaging, Apr 2019, Venice, Italy. hal-01988262

\section{HAL Id: hal-01988262 \\ https://hal.inria.fr/hal-01988262}

Submitted on 21 Jan 2019

HAL is a multi-disciplinary open access archive for the deposit and dissemination of scientific research documents, whether they are published or not. The documents may come from teaching and research institutions in France or abroad, or from public or private research centers.
L'archive ouverte pluridisciplinaire HAL, est destinée au dépôt et à la diffusion de documents scientifiques de niveau recherche, publiés ou non, émanant des établissements d'enseignement et de recherche français ou étrangers, des laboratoires publics ou privés. 


\title{
Analytical Fiber ODF Reconstruction in 3D Polarized Light Imaging: Performance Assessment
}

\author{
A. Alimi ${ }^{\dagger}$, S. Deslauriers-Gauthier ${ }^{\dagger}$, F. Matuschke ${ }^{\star}$, D. Schmitz ${ }^{\star}$, M. Axer ${ }^{\star}$ and R. Deriche D $^{\dagger \S}$ \\ ${ }^{\dagger}$ Athena Project-Team, Inria Sophia Antipolis-Méditerranée, Université Côte d'Azur, France \\ * Institute of Neuroscience and Medicine (INM-1), Research Center Jülich, Jülich, Germany
}

\begin{abstract}
Three dimensional Polarized Light Imaging (3D-PLI) allows to map the spatial fiber structure of postmortem tissue at a sub-millimeter resolution, thanks to its birefringence property. Different methods have been recently proposed to reconstruct the fiber orientation distribution function (fODF) from high-resolution vector data provided by 3D-PLI. Here, we focus on the analytical fODF computation approach, which uses the spherical harmonics to represent the fODF and analytically computes the spherical harmonics coefficients via the spherical Fourier transform. This work deals with the assessment of the performance of this approach on rich synthetic data which simulates the geometry of the neuronal fibers and on human brain dataset. A computational complexity and robustness to noise analysis demonstrate the interest and great potential of the approach.
\end{abstract}

Index Terms - fiber orientation distribution function ODF, 3D-PLI, polarized light imaging, analytical method, spherical harmonics, spherical Fourier transform

\section{INTRODUCTION}

Three dimensional Polarized Light Imaging (3D-PLI) is a optical imaging technique that utilizes the birefringence property of postmortem tissue to map its spatial organization at resolution lower than millimeters [2, 3, 4]. It provides high resolution 3D fiber orientation vector data, from which fiber orientation distribution functions can be reconstructed at different spatial scales. This fODF, well known in the diffusion Magnetic Resonance Imaging (dMRI) community [5, 6, 7], is a useful tool for the comparison of 3D-PLI and dMRI fiber orientation estimates, for multi-modality imaging, and for the validation of dMRI results.

Different groups have proposed methods to reconstruct the fODF from 3D-PLI data. Axer et al. (2016) [8] are the

$\S$ This work was partly supported by ANR "MOSIFAH" under ANR13-MONU-0009-01, the ERC under the European Union's Horizon 2020 research and innovation program (ERC Advanced Grant agreement No 694665:CoBCoM). ${ }^{\star}$ This work has also received partially funding from the European Union's Horizon 2020 Research and Innovation Program under Grant Agreement No. 7202070 (Human Brain Project SGA2). * The authors gratefully acknowledge the computing time granted by the JARA-HPC Vergabegremium and provided on the JARA-HPC Partition part of the supercomputer JURECA at Forschungszentrum Jülich [1]. first to introduce the pliODF which uses the concept of supervoxel (SV) to downsample the high resolution data, and then estimate the fODF on the unit sphere by expanding a normalized directional histogram (a discretized distribution of orientations) with a series of spherical harmonics.

Recently, Alimi et al. (2017), first proposed to regularize the pliODF using the Laplace-Beltrami operator [9], before introducing an analytical approach [10] to compute the fODF from 3D-PLI data. This latter method is based on elegantly defining each fiber orientation in a native voxel as a 2D Dirac delta function on the unit sphere and the fODF as a weighted sum of these Diracs within each super-voxel. As in [8], we refer to a tissue voxel containing a single high-resolution fiber orientation as 'native voxel'. The super-voxel regroups $K=$ rows $\times$ columns $\times$ slices native voxels.

The purpose of the paper is to study the performance of the analytical fiber ODF reconstruction method [10] through highly rich synthetic dataset which simulates the brain white matter fibers using a geometric model, and through human brain dataset. In the following, the analytical fiber ODF approach implementation is briefly presented as well as the synthetic data generation method, then the different performed tests are described and finally the results are discussed in the last section.

\section{METHODS}

\subsection{Fiber ODF reconstruction implementation}

The analytical approach makes use of a truncated spherical harmonics series $Y_{l}^{m}$ to represent in a continuous domain the fiber orientation distribution functions in a given super-voxel as

$$
f(\theta, \phi) \approx \sum_{l=0}^{L_{\max }} \sum_{m=-l}^{l} c_{l m} Y_{l}^{m}(\theta, \phi)
$$

where $L_{\max }$ is the maximum order or bandlimit of the truncation, $c_{l m}$ are the SH coefficients which completely describe the fODF [10] and $(\theta, \phi)$ are the spherical coordinates of a fiber orientation vector in a given direction.

This fiber ODF $f$ is modeled as a sum of 2D Dirac delta $\delta$ functions. Each $\delta$ function represents the spatial orientation of a single fiber orientation vector on the unit sphere. 


\begin{tabular}{cccc}
\hline & \multicolumn{2}{c}{ Super-voxel size } \\
\cline { 2 - 4 }$L_{\max }$ & $50 \times 50 \times 1$ & $10 \times 10 \times 1$ & $5 \times 5 \times 1$ \\
\hline 4 & $0: 04.14$ & $0: 05.53$ & $0: 09.02$ \\
6 & $0: 08.86$ & $0: 10.49$ & $0: 14.50$ \\
8 & $0: 15.98$ & $0: 18.04$ & $0: 22.13$ \\
10 & $0: 26.05$ & $0: 27.68$ & $0: 32.45$ \\
12 & $0: 39.15$ & $0: 40.64$ & $0: 44.99$ \\
\hline
\end{tabular}

Table 1. Computational running times in minutes:seconds. The ROI consists of a human brain coronal slice of $1000 \times$ $1700 \times 1$ native voxels.

Since 3D-PLI provides us with all the $K$ fiber orientations $\left(\theta_{k}, \phi_{k}\right)$ located in the super-voxel, the corresponding fODF is modelled as $f(\theta, \phi)=\frac{1}{K} \sum_{k=1}^{K} \delta\left(\cos \theta-\cos \theta_{k}\right) \delta\left(\phi-\phi_{k}\right)$ and the $c_{l m}$ coefficients are analytically computed through the spherical Fourier transform,

$$
c_{l m}=\frac{1}{K} \sum_{k=1}^{K} \overline{Y_{l}^{m}}\left(\theta_{k}, \phi_{k}\right) .
$$

Although this approach uses the $\mathrm{SH}$ to represent the fODF, it is independent of the SH basis used and here the real and symmetric basis defined in [6] is considered. In this case, the number of terms of the SH basis of order $l$ is $N=(1 / 2)(l+1)(l+2)$, where only the even orders are chosen.

\subsection{Synthetic data generation}

For the simulation of the 3D-PLI datasets, two 3D fiber bundles intersecting at an angle $\alpha$ are generated. A 3D collision check is performed to ensure that the individual fibers do not overlap. For this purpose, the fibers are divided into short segments so that two segments can be checked for a collision. In the event of a collision, the two segments are slowly pushed apart. This happens simultaneously for all colliding objects and is repeated until all segments have no more collisions.

The 3D models generated in this way are then used for the simulation using SimPLI tool [11, 12] to produce synthetic 3D-PLI datasets. The noise of the CCD camera is modelled by a negative binomial distribution in which the variance $\sigma$ depends on the expected light intensity $\mu$ by $\sigma=3 \mu$ as in [13]. From the generated 3D-PLI signal, high-resolution orientations are reconstructed based on the approaches in [3, 13].

\subsection{Human data description}

The processed experimental data was taken from a human brain. It consists in one coronal section of the right hemisphere out of the dataset described in [13]. The section has a thickness of $70 \mu \mathrm{m}$ which was measured with a pixel size of $64 \times 64 \mu \mathrm{m}^{2}$, analyzed pixel-wise for its fiber orientations and registered onto an image of the brain block before sectioning.

\begin{tabular}{ccc}
\hline$L_{\max }$ & Angular Limit & Angular Error \\
\hline 4 & $52^{\circ}$ & $10.18^{\circ}$ \\
6 & $37^{\circ}$ & $8.11^{\circ}$ \\
8 & $29^{\circ}$ & $5.47^{\circ}$ \\
10 & $25^{\circ}$ & $3.89^{\circ}$ \\
12 & $21^{\circ}$ & $1.55^{\circ}$ \\
\hline
\end{tabular}

Table 2. Angular resolution limit improves with $L_{\max }$ while angular error is reduced.

\subsection{Performance assessment}

To assess the performance of our fiber ODF reconstruction approach, we carry out tests on synthetic data as well as on experimental human brain 3D-PLI datasets.

Computational complexity analysis Let us assume, in each super-voxel, there are $K=X Y Z$ native voxels. We recall our method uses SH representation and $N$ is the number of SH coefficients which describe the fiber ODF. Using the Diracs, the computation of each SH coefficient is the simple averaged sum from Equation 2, therefore its reconstruction is $\mathcal{O}(N)$. This test is performed on the previously described coronal brain slice from the right hemisphere.

Peaks detection and Angular resolution In dMRI community it is generally assumed that the local maxima or peaks of the normalized fODF coincide with the fiber directions [5, 6]. The same assumption is considered here in order to estimate the fODF local orientations and compare with ground truth. To this end, we generate noise-free synthetic data, consisting of two fiber bundles crossing at different angles $\alpha$ ranging from $0^{\circ}$ to $90^{\circ}$ in $1^{\circ}$ increments. Therefore, we can quantitatively study the angular resolution limitations of the reconstructed fODF. Here, the angular resolution limit is the critical angle under which the peaks of the two fiber populations are confounded, that is, they can not be detected separately.

Noise robustness Noise robustness is actually assessed during fiber orientation estimation stage in 3D-PLI analysis work-flow [4, 14, 15, 13], prior to fODF reconstruction. That is, the fODF reconstruction method does not directly deal with the noise (removal). However, it is still crucial to test our algorithm's robustness to noise. Therefore, we perform a synthetic experiment on noisy dataset (see 2.2) consisting of two fiber populations crossing at angles $\alpha$ varying from $10^{\circ}$ to $90^{\circ}$ in $10^{\circ}$ increments. Such noisy dataset is generated a 100 times and the algorithm is run each time to estimate the angular resolution limit.

Angular error In these previous tests, we also evaluate the fODF angular error as $\beta=\frac{180}{\pi M} \sum_{m=1}^{M} \arccos \left(\mathbf{v}_{m}^{t} \mathbf{w}_{m}\right)$ which is the average distance between $\mathbf{v}_{m}$ the $m^{t h}$ ground truth orientation and $\mathbf{w}_{m}$ the closest of the recovered peak orientations of the fiber ODF and $M$ is the number of fibers in the super-voxel. 


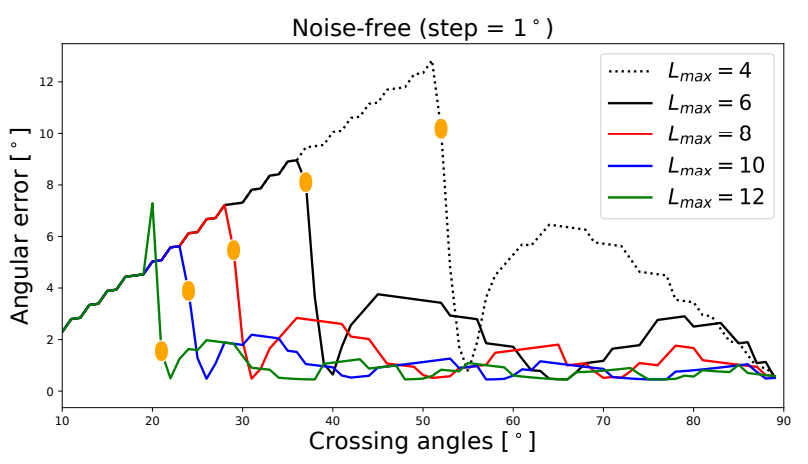

Fig. 1. Angular error from noise-free data.

\section{RESULTS AND DISCUSSION}

Computational running time We study the fODF reconstruction speed of our method on a 4 processors, $2.60 \mathrm{GHz}(\times 4)$, 16 GB RAM machine, and Table 1 shows the results. As expected, the analytical approach is very fast. This is due to the choice of defining each fiber orientation as a 2D Dirac function on the unit sphere, which simplifies and fastens the computation of the SH coefficients, analytically. Notice how fast and computationally efficient is our approach and that the computation time decreases when the super-voxel size increases as observed in [8].

Angular resolution Table 2 shows the results of the determination of the critical angle of our analytical fODF reconstruction method in a noise-free setting. The angular resolution is generally high, going from $51^{\circ}$ down to $21^{\circ}$ when $L_{\max }$ goes from 4 to 12 , while the angular error improves of roughly $10^{\circ}$. This is because the higher order frequencies are taken into account in the fODF estimation when SH bandlimit increases and thanks to the use of the Diracs. This result is backed up in Figure 1 where the angular error is plotted against the crossing angles. It shows the improvement of both the critical angle, spotted in orange dots, and the angular error, for every $L_{\max }$, in good agreement with results previously shown in [10].

Noise robustness Table 3 gives the angular resolution limit $\alpha$ and the angular error of the reconstructed fODF from noisy data. Results are indicated in intervals since the considered crossing angles range from $10^{\circ}$ to $90^{\circ}$ in $10^{\circ}$ increments. We can see that, for $L_{\max } \leq 6, \alpha$ is roughly between $40^{\circ}$ to $60^{\circ}$ as in the noise-free case and between $30^{\circ}$ to $40^{\circ}$ when $L_{\max }$ is higher, indicating thus not a big influence of the noise on the method, which therefore recovers successfully the fODF peaks beyond these upper limits with respect to $L_{\max }$. Interpreting the angular error $\beta$, however, is more tricky. Indeed, unlike in the noiseless case where $\alpha$ is incremented of $1^{\circ}$, in the noisy setting, the increment is $10^{\circ}$ and $\beta$ is less precisely estimated and falls into intervals as displayed in Figure 2. Nevertheless, we can see that when the fODF peaks are

\begin{tabular}{ccc}
\hline$L_{\max }$ & Angular Limit & Angular Error \\
\hline 4 & {$\left[50^{\circ}, 60^{\circ}\right]$} & {$\left[3^{\circ}, 12^{\circ}\right]$} \\
6 & {$\left[40^{\circ}, 50^{\circ}\right]$} & {$\left[1^{\circ}, 10^{\circ}\right]$} \\
8 & {$\left[30^{\circ}, 40^{\circ}\right]$} & {$\left[3^{\circ}, 7^{\circ}\right]$} \\
10 & {$\left[30^{\circ}, 40^{\circ}\right]$} & {$\left[2^{\circ}, 7^{\circ}\right]$} \\
12 & {$\left[30^{\circ}, 40^{\circ}\right]$} & {$\left[3^{\circ}, 10^{\circ}\right]$} \\
\hline
\end{tabular}

Table 3. Angular resolution limit in noisy setting.

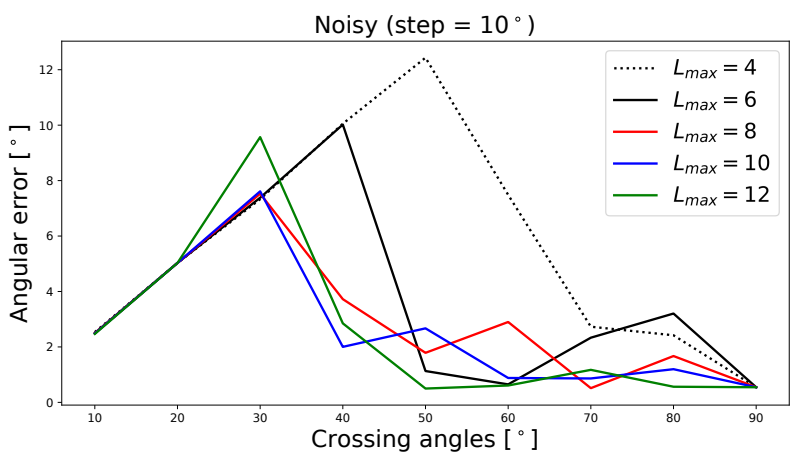

Fig. 2. Angular error from noisy data.

successfully detected, the angular error is yet less than $13^{\circ}$ even at the lowest $L_{\max }=4$.

On human brain data The coronal brain slice, acquired and described in [13], was used to perform the computational running time test, and here, to illustrate reconstructed fODFs in Figure 3 at different spatial scales, by varying the SV size. This data consists of $1350 \times 1950 \times 1$ native voxels. The region of interest (ROI) in yellow displays the stratum sagittale in deep white matter while the one in green shows fibers fanning into the cortex. In both ROIs, where the fiber structure is well known, it can be observed that the anatomical organization is still conserved at different spatial resolutions. This demonstrates that the analytical approach preserves the integrity of the fiber architecture in the brain.

Note that in [10] we show the analytical approach outperforms both pliODF and its regularized version however further comparisons could be carried out in future work.

\section{CONCLUSION}

In this paper, we assess the performance of the analytical fiber orientation distribution functions reconstruction method in 3D Polarized Light Imaging using both highly rich synthetic and human brain datasets, with considerable findings. First, the fODF is analytically described on a spherical harmonics basis and elegantly and efficiently computed via the spherical Fourier transform and by means of the Diracs. This makes the fODF computationally very efficient, very fast and less prone to angular error. Second, the recovered fODF angu- 


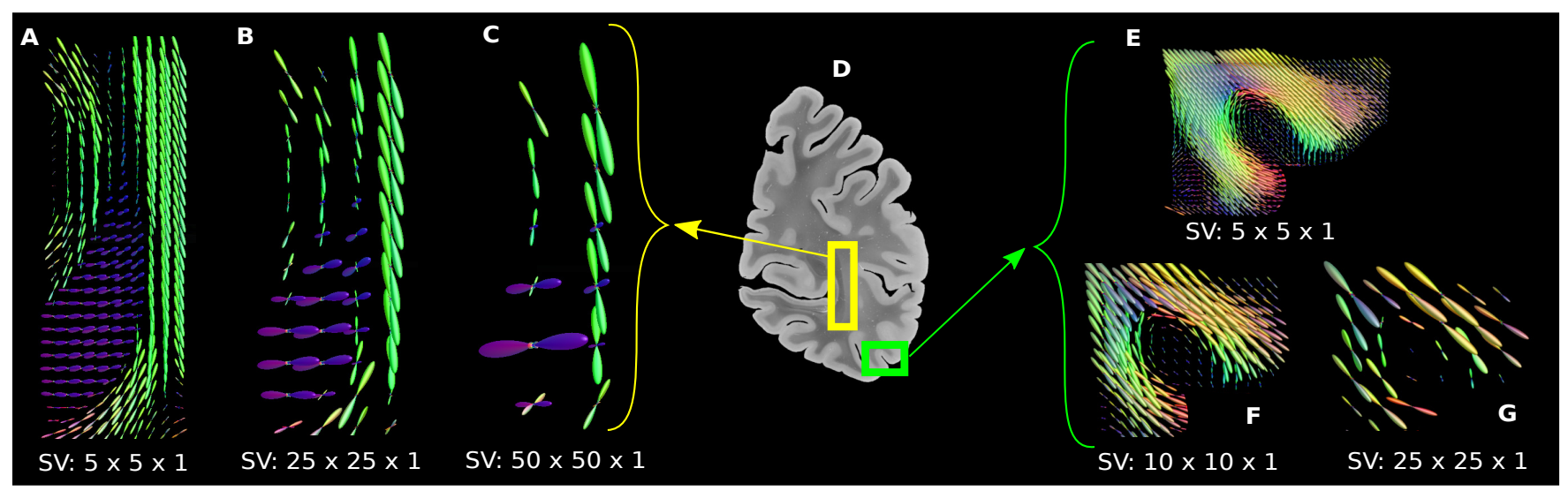

Fig. 3. Fiber ODFs computed from a coronal slice of the brain's right hemisphere.

lar resolution limit is high enough to allow resolving crossings even with very acute angles. Third, tests on noisy datasets show very little influence of the noise on both the angular limit and the angular error of the reconstructed fiber ODF, revealing thus that our method is robust to noise. Finally, our algorithm preserves, at different spatial scales, the integrity of the fiber architecture in the human brain.

\section{REFERENCES}

[1] Krause et al., "Jureca: Modular supercomputer at jülich supercomputing centre," Journal of large-scale research facilities JLSRF, vol. 4, pp. 132, 2018.

[2] Jouk et al., "Three-dimensional cartography of the pattern of the myofibres in the second trimester fetal human heart," Anatomy and embryology, vol. 202, no. 2, pp. 103-118, 2000 .

[3] Axer et al., "A novel approach to the human connectome: ultra-high resolution mapping of fiber tracts in the brain," NI, vol. 54, no. 2, pp. 1091-1101, 2011.

[4] Alimi et al., "Solving the inclination sign ambiguity in three dimensional polarized light imaging with a pdebased method," in Biomedical Imaging (ISBI 2017), 2017 IEEE 14th International Symposium on. IEEE, 2017, pp. 737-740.

[5] Tuch, "Q-ball imaging," Magnetic resonance in medicine, vol. 52, no. 6, pp. 1358-1372, 2004.

[6] Descoteaux et al., "Regularized, fast, and robust analytical Q-ball imaging," Magnetic resonance in medicine, vol. 58, no. 3, pp. 497-510, 2007.

[7] Aganj et al., "Reconstruction of the orientation distribution function in single-and multiple-shell q-ball imaging within constant solid angle," Magnetic Resonance in Medicine, vol. 64, no. 2, pp. 554-566, 2010.
[8] Axer et al., "Estimating fiber orientation distribution functions in 3D-polarized light imaging," Frontiers in neuroanatomy, vol. 10, 2016.

[9] Alimi et al., "Regularizing the ODF estimate with the Laplace-Beltrami operator in 3D Polarized Light Imaging," CoBCoM 2017 - Computational Brain Connectivity Mapping Winter School Workshop, Nov. 2017.

[10] Alimi et al., "An analytical fiber odf reconstruction in 3d polarized light imaging," in Biomedical Imaging (ISBI 2018), 2018 IEEE 15th International Symposium on. IEEE, 2018, pp. 1276-1279.

[11] Dohmen et al., "Understanding fiber mixture by simulation in 3d polarized light imaging," NeuroImage, vol. 111, pp. 464-475, 2015.

[12] Menzel et al., "A jones matrix formalism for simulating three-dimensional polarized light imaging of brain tissue," Journal of The Royal Society Interface, vol. 12, no. 111, pp. 20150734, 2015.

[13] Schmitz et al., "Derivation of fiber orientations from oblique views through human brain sections in $3 \mathrm{~d}-$ polarized light imaging," Frontiers in Neuroanatomy, vol. 12, pp. 75, 2018.

[14] Kleiner et al., "Classification of ambiguous nerve fiber orientations in 3d polarized light imaging," in International Conference on Medical Image Computing and Computer-Assisted Intervention. Springer, 2012, pp. 206-213.

[15] Wiese et al., "Polarized light imaging of the human brain: a new approach to the data analysis of tilted sections," in Polarization: Measurement, Analysis, and Remote Sensing XI. International Society for Optics and Photonics, 2014, vol. 9099, p. 90990U. 